CLINICAL STUDY

\title{
Persistence at 1 year of oral antiosteoporotic drugs: a prospective study in a comprehensive health insurance database
}

\author{
Cyrille B Confavreux $^{1}$, Florence Canoui-Poitrine ${ }^{2,3,4}$, Anne-Marie Schott ${ }^{1,2}$, Véronique Ambrosi ${ }^{5}$, \\ Valérie Tainturier ${ }^{5}$ and Roland D Chapurlat ${ }^{1}$ \\ ${ }^{1}$ INSERM UMR 1033 - Université de Lyon, Department of Rheumatology, Hôpital Edouard Herriot, Pavillon F, Hospices Civils de Lyon, 5 Place \\ d'Arsonval, 69003 Lyon, France, ${ }^{2}$ Pôle Information Médicale Evaluation Recherche, Hospices Civils de Lyon, Université de Lyon, RECIF, EA 2149, \\ 69003 Lyon, France, ${ }^{3}$ APHP, Hôpital Henri Mondor, Service de Santé Publique, F-94000 Créteil, France, ${ }^{4}$ Laboratoire d'Investigation Clinique, Université \\ Paris Est, EA 4393, F-94000 Créteil, France and ${ }^{5}$ Direction Régionale du Service Médical de l'Assurance Maladie, 69003 Lyon, France
}

(Correspondence should be addressed to C B Confavreux; Email: cyril.confavreux@inserm.fr)

\begin{abstract}
Objective: Treatments against osteoporosis have demonstrated fracture risk reduction but persistence to therapy remains a major issue. Intermittent regimens have been developed to improve persistence. The aim of this 1-year prospective study was to compare, in the general population, the persistence of various oral regimens of antiosteoporotic treatment.

Methods: We conducted this prospective study in the French comprehensive public health insurance database of the Rhône-Alpes region. Women aged 45 years or older who had a first reimbursement of an oral antiosteoporotic treatment during February 2007 composed the study cohort. Persistence was defined by the proportion of patients refilling a prescription in the pharmacist delivery register (ERASME). Using statistical analyses like Kaplan-Meier survival curves and log-rank tests, we compared the treatment persistence of strontium ranelate, raloxifene, and daily-, weekly-, and monthly bisphosphonates.

Results: Two thousand four hundred and nineteen patients were included over a period of 1 month and followed up for 12 months. Two hundred and eighty-nine (11.9\%) patients were treated with monthly bisphosphonates, 1298 (53.7\%) with weekly bisphosphonates, and $832(34.4 \%)$ with daily treatments (526 strontium ranelate $(21.7 \%), 296$ raloxifene (12.2\%), and 10 bisphosphonates $(0.4 \%)$ ). At 1 year, overall persistence was $34 \%$. Fifty percent of patients on monthly bisphosphonates were still persistent while only $37 \%$ of patients on weekly bisphosphonates, $34 \%$ on raloxifene, and $16 \%$ on strontium ranelate were persistent. Therapy monitoring with biochemical markers or bone mineral density was associated with improved persistence.

Conclusion: Overall persistence at 1 year was low, but intermittent regimens were associated with higher persistence rates, along with women who had therapy monitoring.
\end{abstract}

European Journal of Endocrinology 166 735-741

\section{Introduction}

Several classes of oral drugs can be used in the treatment of postmenopausal osteoporosis. They have all shown efficacy to reduce fracture risk. Adherence to the treatment, however, remains a critical issue in the management of chronic diseases such as osteoporosis. One reason is that patients do not perceive the need for treatment because osteoporosis is an asymptomatic disease before the first fracture $(1,2)$. Therapeutic adherence is the extent to which a person takes medication under the physician's recommendation. Adherence includes persistence, length of time the patient continues taking medication from initiation, and compliance, which describes how regimen and doses are taken. Compliance is commonly evaluated by the medication possession rate (MPR) corresponding to the number of pills correctly taken divided by the number of pills prescribed. A good compliance is generally above $80 \%$.

In osteoporosis, therapeutic adherence is logically associated with fracture risk reduction (3). Independent correlation of persistence with fracture risk reduction was also observed in two US claim databases including 35537 women receiving bisphosphonates. Only $43 \%$ of patients were refill compliant and $20 \%$ persisted after 2 years of follow-up (4). In the PHARMO-RLS study, adherence to bisphosphonate therapy was evaluated through compliance (MPR) in new postmenopausal female bisphosphonate users. During the follow-up, a steady increase in noncompliant patients (MPR <80\%) was observed: $42 \%$ after 1 year, $51 \%$ after 2 years, and $60 \%$ after 3 years. Noncompliant bisphosphonate use was associated with a $40 \%$ increased risk of osteoporotic 
fracture requiring hospitalization (5). In another observational study of 11252 postmenopausal women extracted from a drug dispensation database, an antiosteoporotic medication covering at least $80 \%$ of the time was independently associated with a fracture risk reduction of $16 \%(6)$. In the UK, the General Practice Research Database (GPRD) 1-year persistence rate for risedronate or alendronate was $58 \%$ but decreased to $24 \%$ after 5 years. Persistence of at least 24 months showed a hip fracture risk reduction of $34 \%$, and hip fracture hazard rates diverged after 1 year of treatment (7). The study conducted among the new postmenopausal female alendronate users of the Belgian national social security database showed a low global persistence $(40 \%)$ at 12 months, but persistent patients had a $60 \%$ hip fracture relative risk reduction. MPR was also a significant predictor of hip fracture with an increased adjusted risk of $0.4 \%$ for each $1 \%$ decrease in the MPR. MPR was significantly higher for patients who received weekly alendronate $(70.5 \%)$ compared with daily regimen $(58.6 \%)$, raising the issue of optimal regimen (8). Similarly, in a US claim database, a $42 \%$ persistence rate was observed with alendronate after 24 months of follow-up. Patients with weekly regimen were more likely to be persistent $(49 \%)$ than nonpersistent (38\%) (9).

All these studies, however, have not been conducted in the general population. Some of them used private insurance claim databases and some used regrouping of general practitioners (GPs). In addition, all these studies were retrospective, so potential predictors of adherence could not be ascertained before starting therapy. Thus, we have conducted a prospective study to assess the persistence of several antiosteoporotic drugs and whether monthly regimen was associated with a better persistence in a universal coverage health care system database providing data of the general population.

\section{Materials and methods}

\section{Database}

The Rhône-Alpes area has six million inhabitants representing $\sim 10 \%$ of the French population. We have prospectively used the Rhone-Alpes ERASME database (Extraction, Recherche et Analyse pour un Suivi Médico-Économique), which means Extraction, Research and Analysis of a Medico-Economical followup. This database is run by the French public social insurance (Sécurité Sociale). The ERASME database contains fee-for-service claims for in- and outpatient medical services supplied to $80 \%$ of the residents of France, as well as all drugs and tests that are ordered by physicians for community-dwelling patients. The remaining $20 \%$ of the French residents are covered by special subdivisions of the French social health care system, depending on their job. Before being nationally centralized, claims are first centralized at the regional level by each regional center of the CNAMTS (Caisse Nationale d'Assurance Maladie des Travailleurs Salariés). The organization of data collection and the database has been previously described in detail (10). We created an inception cohort of all women aged 45 years or older living in the Rhône-Alpes area with a first reimbursement of an oral antiosteoporotic treatment (i.e. raloxifene, strontium ranelate, and daily-, weekly-, and monthly brand and generic bisphosphonates) between 1st and 28th February 2007. Patients treated with teriparatide or intravenous bisphosphonate were excluded. Patients previously treated with any antiosteoporotic drugs including teriparatide or intravenous bisphosphonates were also excluded.

\section{Inclusion data}

We used the date of first reimbursement as the date of entry, and we collected for each woman the type of antiosteoporotic treatment with raloxifene, strontium ranelate, daily-, weekly-, and monthly brand and generic bisphosphonates; age; presence of severe chronic comorbidity; and prior bone mineral density (BMD) reimbursement. For the definition of chronic severe comorbidity, we used the ALD (Affection de Longue Durée or Chronic Severe Comorbidity (SCC)) status as a proxy. The ALD status is given to patients who have chronic diseases, e.g. diabetes, cancer, cardiac failure, renal deficiency, rheumatoid arthritis, and multiple sclerosis. It is considered that these diseases imply costly and long-term treatments and monitoring so that the patients have no co-payment.

\section{Follow-up}

Each woman in the cohort was then followed for 1 year for reimbursement of oral antiosteoporotic treatment, including the number of delivered pills, through record linkage in the Rhone-Alpes health insurance database. Using the number of delivered pills and the treatment regimen (monthly, weekly, and daily), assuming that all delivered pills were effectively taken by the patients, we computed the time to end of refilling in days. For example, a patient delivered 12 pills of a weekly bisphosphonate has a time to end of refilling of 84 days. In the survival analysis, we used a persistence of 365 days, corresponding to a $100 \%$ refilling treatment after 1 year of follow-up. The outcome was time to end of refilling. No refill gap was allowed. Reimbursement of serum type I collagen breakdown product (CTX) assessment 3-6 months after first reimbursement of a drug was also collected during the follow-up to know whether it could be associated with improved persistence.

\section{Statistical analysis}

Categorical variables were presented in $n(\%)$ and continuous variables in mean (S.D.) or median 
(interquartile range (IQR)) as appropriate. Survival time was defined as the time between study entry (i.e. date of first reimbursement of oral antiosteoporotic treatment between 1st and 28th February 2007) and either date of end of refilling before 28th February 2008 or 28th February 2008 as date of the end of the follow-up, which serves as the censoring time point. Kaplan-Meier curves were used to visualize persistence probabilities, with step down when a treatment has been stopped. Kaplan-Meier curves stratified on oral antiosteoporotic treatment were plotted. Comparison of persistence between oral antiosteoporotic treatments was done using a log-rank test. For the estimation of hazard ratios of antiosteoporotic treatment, a multivariate Cox proportional hazard model was fitted including potential confounders, i.e. quartiles of age, severe chronic comorbidity, prior BMD, and serum CTX assessment. We also compared duration of treatment among the various regimens using uni- and multivariate linear regression models. All comparisons were two sided and a $P$ value of $<0.05$ was used to indicate a statistically significant difference. Analyses were performed using STATA 9.1 Software version (StataCorp, College Station, TX, USA) and SPSS 15.0 Software version (SPSS, Inc., Chicago, IL, USA).

\section{Results}

\section{Baseline characteristics}

Two thousand four hundred and nineteen women aged 45 years or older were included over a period of 1 month in February 2007 when they were given for the first time an oral antiosteoporotic treatment. The mean $( \pm$ s.D. $)$ age was 67.7 years $( \pm 10.5)$ and $36.9 \%$ $(n=892)$ of the sample had an associated severe chronic comorbidity.

Interestingly, 897 (37.1\%) of the population underwent a BMD testing in the 6 months preceding the initial drug delivery and $172(7.1 \%)$ had a serum CTX assessment after prescription. Among patients with BMD testing, 35.2\% did not have any CTX assessment, and among patients with CTX assessment, 38.4\% did not have any BMD testing. Thus, patients with BMD testing and patients with serum CTX assessment cannot be considered to be the same population.

Among the 2419 women, 1298 (53.7\%) received a weekly bisphosphonate, $526(21.7 \%)$ strontium ranelate, 296 (12.2\%) raloxifene, and 289 (11.9\%) monthly bisphosphonates. Only some of the patients received a daily bisphosphonate $(n=10 ; 0.4 \%)$. As expected, patients receiving raloxifene were significantly younger $(60.2 \pm 7.8$ years) with less severe chronic comorbidity $(17.2 \%)$ in comparison with the other groups (Table 1).

The median duration of therapy was 168 (IQR: 56-336) days with strontium ranelate and 224 (IQR: 184-365) days with raloxifene. For bisphosphonates, the median significantly increased with the dosing frequency: 84 (IQR: 28-140) days for daily, 308 (IQR: 112-365) for weekly, and 365 (IQR: 180-365) for the monthly regimen (Table 2 ).

\section{One-year persistence and factors associated with persistence}

The overall persistence was 34\% $(n=818 / 2419)$ and varied significantly according to the drug and the regimen (log-rank test $P<0.001$; Fig. 1). Daily treatments had the smallest persistence rates ranging from $10 \%$ on daily bisphosphonates to $16 \%$ on strontium ranelate and $34 \%$ on raloxifene. Monthly bisphosphonates $(50 \%)$ had a better persistence rate than the weekly bisphosphonates (37\%).

In a multivariate analysis (Cox proportional hazard model) including age, severe chronic comorbidity, prior BMD testing, and serum CTX assessment after prescription as covariates, treatment regimen remained independently associated with persistence. Compared with weekly bisphosphonates taken as the reference category, monthly bisphosphonates (hazard ratio $(\mathrm{HR})=1.32$ (1.10; 1.57), $P=0.002$ ) were independently associated with improved persistence. The three daily treatments were independently associated with lower persistence (Fig. 2A). The same results were observed for a persistence defined as $80 \%$ (292 days) of treatment refilling at 1 year (data not shown).

In univariate analysis, patients who underwent CTX measurement (log-rank $P=0.009$; Fig. 3A) or BMD

Table 1 Baseline characteristics of women according to their regimen of antiosteoporotic treatment. Seven hundred and ninety-one (32.7\%) patients underwent BMD measurement and 106 (4.4\%) patients underwent BMD and biochemical marker assessment. Only 66 (2.7\%) patients had a biochemical marker. The remaining $1456(60.2 \%)$ patients had no BMD and no biochemical marker. Continuous variables were expressed as mean (S.D.) and categorical variables as $n(\%)$.

\begin{tabular}{|c|c|c|c|c|c|c|c|}
\hline & Total & Raloxifene & SR & Daily BP & Weekly BP & Monthly BP & $P^{a}$ \\
\hline$n(\%)$ & $2419(100)$ & 296 (12.2) & $526(21.7)$ & $10(0.4)$ & 1298 (53.7) & 289 (11.9) & \\
\hline Age (years) & $67.7(10.5)$ & $60.2(7.8)$ & $68.6(11.1)$ & $65.3(12.5)$ & $69.0(10.4)$ & $68.0(9.6)$ & $<0.001$ \\
\hline SCC & $892(36.9)$ & $51(17.2)$ & $186(35.4)$ & $3(30.0)$ & $541(41.7)$ & $111(38.4)$ & $<0.001$ \\
\hline Prior BMD testing & $897(37.1)$ & $126(42.6)$ & $193(36.7)$ & $2(20.0)$ & 445 (34.3) & $131(45.3)$ & 0.001 \\
\hline CTX testing & $172(7.1)$ & $29(9.8)$ & $44(8.4)$ & $0(0)$ & $77(5.9)$ & $22(7.6)$ & 0.1 \\
\hline
\end{tabular}

${ }^{a}$ ANOVA for continuous variables; Fisher exact test for categorical variables.

SCC, severe chronic comorbidity; SR, strontium ranelate 
Table 2 Comparison of the duration of various regimens of oral antiosteoporotic treatment. Coefficients express the mean duration that patients persisted on therapy in comparison with the reference group (weekly bisphosphonates). They represent the number of additional days on therapy (monthly bisphosphonates) or the number of fewer days on therapy (raloxifene, strontium ranelate, and daily bisphosphonates), compared with weekly regimens. Data are expressed in days.

\begin{tabular}{|c|c|c|c|c|c|}
\hline & Median (Q1-Q3) & Coefficient $(95 \% \mathrm{CI})^{\mathrm{a}}$ & $P$ & Coefficient $(95 \% \mathrm{Cl})^{\mathrm{b}}$ & $\boldsymbol{P}$ \\
\hline Raloxifene & $224(184-365)$ & $-25.7(-41.9--9.6)$ & 0.002 & $-31.5(-48.2-14.8)$ & $<0.001$ \\
\hline SR & $168(56-336)$ & $-57.8(-70.7--44.8)$ & $<0.01$ & $-59.6(-72.5--46.7)$ & $<0.001$ \\
\hline Daily BP & $84(28-140)$ & $-132.5(-212.0--52.9)$ & 0.001 & $-128.9(-208.0--49.8)$ & $<0.01$ \\
\hline Weekly BP & $308(112-365)$ & Reference & - & - & - \\
\hline Monthly BP & $365(180-365)$ & $24.0(7.7-40.3)$ & 0.004 & $20.4(4.2-36.7)$ & 0.014 \\
\hline
\end{tabular}

Q1, first quartile; $\mathrm{Q} 3$, third quartile; $\mathrm{Cl}$, confidence interval; $\mathrm{BP}$, bisphosphonate; SR, strontium ranelate.

aUnivariate linear regression.

${ }^{\mathrm{b}}$ Multivariate linear regression adjusted for age, severe chronic comorbidity, prior BMD testing, and CTX assessment.

evaluation before treatment initiation (log-rank $P<0.001$; Fig. 3B) had a significantly improved persistence. In our multivariate model including the previous covariates (i.e. taking BMD into account), CTX measurement remained significantly associated with persistence with an HR of 1.19 (1.04; 1.37, P<0.05), indicating that CTX, in this population, was an independent predictor of adherence. Prior BMD testing was also an independent predictor of persistence $(\mathrm{HR}=$ $1.31(1.18 ; 1.46), P<0.001)$ in multivariate analysis after adjusting for CTX assessment (Fig. 2B).

The second quartile of age (60-68 years; $\mathrm{HR}=1.19$ (1.04; 1.37), $P=0.013$ ) was another factor independently associated with improved persistence. No association was found with severe and/or chronic comorbidity (ALD).

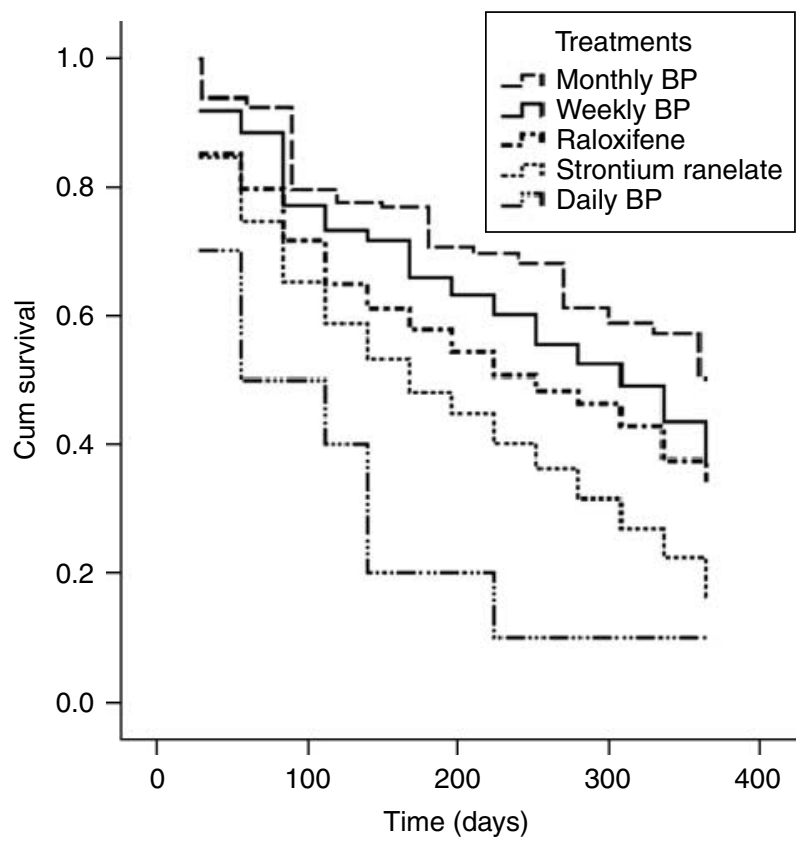

Figure 1 Kaplan-Meier survival curves with time to end of refilling (days) according to the use of initial antiosteoporotic treatment for 1 year: raloxifene, strontium ranelate, and daily-, weekly-, and monthly bisphosphonates (BPs).
In a linear regression analysis we compared duration of refilling for the different regimens. In multivariate analysis adjusted for age, severe chronic comorbidity, prior BMD testing, and serum CTX assessment, duration of treatment with the monthly regimen was on average 20.4 days longer than weekly regimens $(95 \%$ confidence interval (4.2-36.7), $P=0.014)$. The three daily treatment durations were on average (mean) between 31.5 and 128.9 days shorter than weekly treatments (all $P<0.01$; Table 2).

\section{Discussion}

In this prospective study within a comprehensive health insurance database we found that overall persistence at 1 year of all the available oral antiosteoporotic treatments was low. Persistence tended to improve with the longest interval intermittent regimen. Data regarding strontium ranelate have to be interpreted with caution because, during the study period, a European Medicines Agency warning for Drug Reaction with Eosinophilia and Systemic Symptoms (DRESS) was released in November 2007. DRESS refers to a rare adverse reaction occurring usually in the first month of exposure and associating a rash with fever and inflammation of internal organs. If patients continue with the therapy it can lead to death $(11,12,13,14)$. The onset of DRESS, shortly after strontium ranelate marketing, received high media coverage that may have temporarily increased the propensity of patients (or physicians) to stop the drug. This may have also affected treatment initiation but that does not influence our study. Nevertheless, in a recent retrospective study, strontium ranelate persistence at 1 year was also found to be very low at $21.9 \%$ (15).

We observed that persistence improved when BMD testing was carried out done before the initial prescription and when bone markers were measured after 3-6 months of therapy. Observational data have already suggested that BMD testing could increase drug prescription (16) and improve persistence (17). Moreover, patients who thought that their bone density test results did not show osteoporosis are more likely to discontinue the therapy early (2). Results with markers 

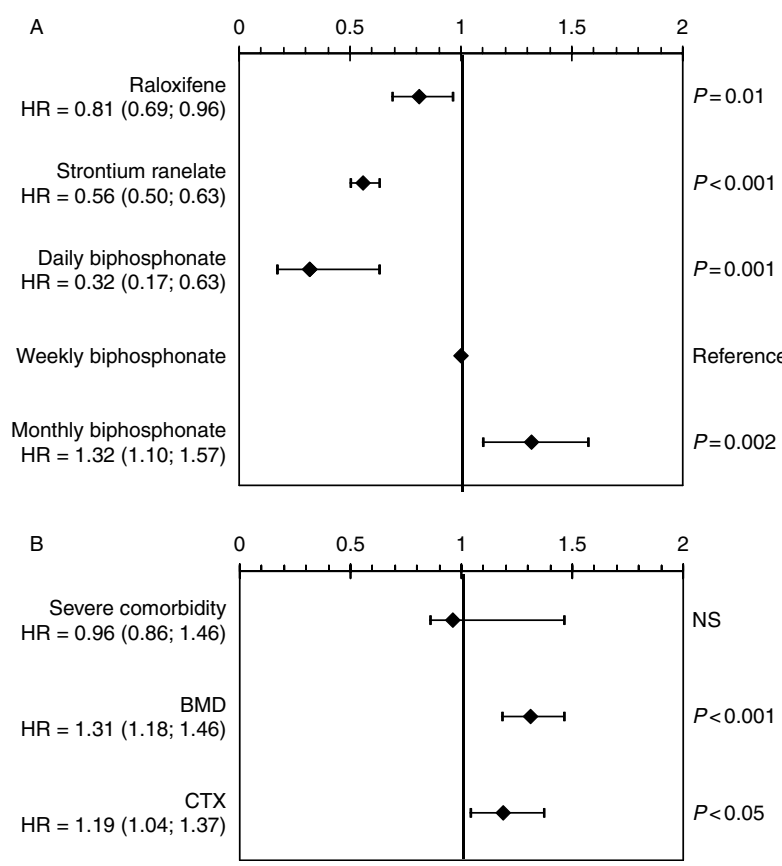

Figure 2 Results of multivariate analysis (Cox proportional hazard model using Wald test) including the following covariates: age, severe chronic comorbidity, prior BMD testing, and serum CTX assessment after prescription. Treatment regimen remained independently associated with persistence (A). BMD testing and CTX assessment were also independent predictors of persistence (B). Filled diamond represents result of the hazard ratio $(\mathrm{HR})$.

are supported by previous data showing that serum CTX assessment after 3-6 months might improve patients' persistence on therapy (18). Monitored patients had a greater adherence than the controlled arm. However, the nature of monitoring did not seem to matter because no difference was observed between the nurse-monitoring and the marker-monitoring groups (18). This study was limited by the small sample size of each group. The IMPACT study analyzed the effect on persistence of monitoring bone turnover markers (uNTX) in postmenopausal women treated with risedronate. A total of 2302 women were randomized between standard care and reinforcement with the marker result. IMPACT reported a surprisingly high 1-year persistence rate in both the groups with $77 \%$ in the standard care group and $80 \%$ in the reinforcement group (19). Nevertheless, the positive feedback message to the patient was associated with improved persistence.

We found that intermittent regimens provided better persistence results at 1 year, confirming previous studies comparing daily with weekly regimens $(8,20)$. Recently, in a different French prescription database within a network of 1200 GPs around the country (Thales), Cotté et al. (21) have reported a clear superiority of 1-year persistence rate with monthly ibandronate $(47 \%)$ in comparison with weekly bisphosphonates $(30 \%)$. One limitation of the Thales database is that it does not represent all prescribers and all patients. Indeed, it covers a set of GPs who are paid to participate in the network and may have greater implications for patients. Another potential bias is the absence of possibility for identifying patients who switch to out-of-network physicians. In contrast, our study cohort has the advantage of being representative of the general population. Even when patients switched to another physician, their reimbursements are still managed by the public health insurance. Interestingly, Thales network also showed that densitometry performed before treatment initiation improved adherence. The benefit of monthly regimen is sustained by the results from the PERSIST study, which randomized patients into weekly vs monthly bisphosphonate groups. Persistence in the monthly bisphosphonate group associated with a patient support program (PSP) was superior (57 vs 39\%). Nevertheless, the follow-up was short (6 months) and there was no monthly arm without PSP (22). Thus, it remained difficult to interpret
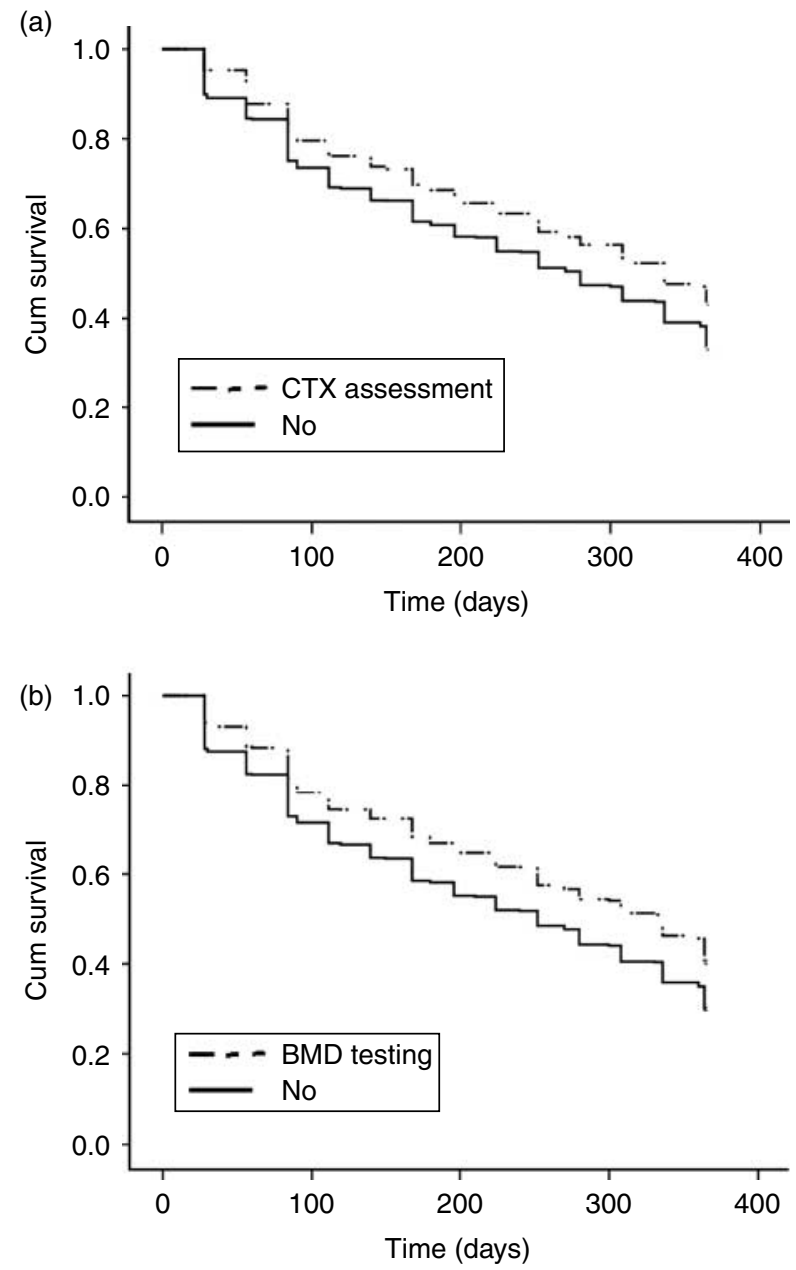

Figure 3 Kaplan-Meier survival curves with time to end of refilling (days) according to (A) CTX assessment (log-rank test $P=0.009$ ) and (B) BMD testing (log-rank test $P<0.001$ ). 
the relative contributions of the PSP and the dosing regimen. In another study on US retail pharmacy prescription refills, no superiority with monthly regimen was found. However, the study was performed soon after the initial release of ibandronate and results are biased by the refilling gap of 30 days that disadvantaged the monthly regimen and by the co-payment variation in the US healthcare system, with many patients initiating monthly therapy for free with a voucher and not refilling the first box. There was also no information about free samples directly distributed by GPs (23).

Several other determinants of therapeutic adherence have been reported, such as hospitalization for osteoporosis (24), an initial prescription by a rheumatologist (24), patient motivation $(25,26)$, and the onset of bothersome side effects (2). Among the side effects, gastrointestinal problems before or during bisphosphonate therapy have been associated with a decreased persistence of about $20 \%$ at 12 months (17) and reduced compliance $(24)$.

Our study had strengths and weaknesses. It was conducted in a comprehensive health insurance database, so it described a representative sample of the general French population, with almost no loss to followup. Its prospective nature allowed for collection of potential predictors associated with patient adherence. However, it was based on refilling claims so we cannot assert that those drugs that were bought were really used appropriately. The main limitation is the observational nature of the database, which does not provide any reason for interruption or any information on patients, i.e. whether they definitively stopped their treatment or switched to another antiosteoporotic treatment. We also have no indication on prescriber specialty or patients' profiles, such as the severity of osteoporosis, nature of severe comorbidity, association with steroid therapy, vitamin D status, and calcium and vitamin D supplements. Indeed, it is conceivable for instance that patients with more severe osteoporosis tend to have a higher persistence and that patients' profiles may have influenced the medical decision to prescribe the antiosteoporotic treatment. Our study was performed before atypical fractures with long-term bisphosphonate use were reported. It remains unknown whether this recent description has affected the 1-year persistence rate $(27,28)$.

In conclusion, persistence of antiosteoporotic drugs at 1 year in the French general population remained low, even with intermittent regimens which, however, tended to improve the rate of persistence. Supporting the initial prescription with BMD evaluation and early bone marker evaluation also tended to increase persistence.

\section{Declaration of interest}

F Canoui-Poitrine, A-M Schott, V Ambrosi, and V Tainturier have nothing to declare. C B Confavreux received lecture fees from Amgen, Ardix, Lilly, and MSD. R D Chapurlat received lecture fees from Amgen, Lilly, Merck, Novartis, Servier, and Roche.

\section{Funding}

R D Chapurlat received grant support from Amgen, Lilly, Merck, Novartis, Roche, Sanofi-Aventis, Servier, and Warner-Chilcott.

\section{References}

1 Reginster JY, Rabenda V \& Neuprez A. Adherence, patient preference and dosing frequency: understanding the relationship. Bone 200638 S2-S6. (doi:10.1016/j.bone.2006.01.150)

2 Tosteson AN, Grove MR, Hammond CS, Moncur MM, Ray GT, Hebert GM, Pressman AR \& Ettinger B. Early discontinuation of treatment for osteoporosis. American Journal of Medicine 2003115 209-216. (doi:10.1016/S0002-9343(03)00362-0)

3 Huybrechts KF, Ishak KJ \& Caro JJ. Assessment of compliance with osteoporosis treatment and its consequences in a managed care population. Bone 200638 922-928. (doi:10.1016/j.bone.2005. 10.022)

4 Siris ES, Harris ST, Rosen CJ, Barr CE, Arvesen JN, Abbott TA \& Silverman S. Adherence to bisphosphonate therapy and fracture rates in osteoporotic women: relationship to vertebral and nonvertebral fractures from 2 US claims databases. Mayo Clinic Proceedings 200681 1013-1022. (doi:10.4065/81.8.1013)

5 Penning-van Beest FJ, Erkens JA, Olson M \& Herings RM. Loss of treatment benefit due to low compliance with bisphosphonate therapy. Osteoporosis International 200819 511-517. (doi:10. 1007/s00198-007-0466-1)

6 Caro JJ, Ishak KJ, Huybrechts KF, Raggio G \& Naujoks C. The impact of compliance with osteoporosis therapy on fracture rates in actual practice. Osteoporosis International 200415 1003-1008. (doi:10.1007/s00198-004-1652-z)

7 Gallagher AM, Rietbrock S, Olson M \& van Staa TP. Fracture outcomes related to persistence and compliance with oral bisphosphonates. Journal of Bone and Mineral Research 200823 1569-1575. (doi:10.1359/jbmr.080510)

8 Rabenda V, Mertens R, Fabri V, Vanoverloop J, Sumkay F, Vannecke C, Deswaef A, Verpooten GA \& Reginster JY. Adherence to bisphosphonates therapy and hip fracture risk in osteoporotic women. Osteoporosis International 200819 811-818. (doi:10. 1007/s00198-007-0506-x)

9 Gold DT, Martin BC, Frytak JR, Amonkar MM \& Cosman F. A claims database analysis of persistence with alendronate therapy and fracture risk in post-menopausal women with osteoporosis. Current Medical Research and Opinion 200723 585-594. (doi:10. 1185/030079906X167615)

10 Martin-Latry K \& Begaud B. Pharmacoepidemiological research using French reimbursement databases: yes we can! Pharmacoepidemiology and Drug Safety 201019 256-265. (doi:10.1002/ pds.1912)

11 Iyer D, Buggy Y, O'Reilly K \& Searle M. Strontium ranelate as a cause of acute renal failure and dress syndrome. Nephrology 2009 14 624. (doi:10.1111/j.1440-1797.2009.01125.x)

12 Jonville-Bera AP, Crickx B, Aaron L, Hartingh I \& Autret-Leca E. Strontium ranelate-induced DRESS syndrome: first two case reports. Allergy 200964 658-659. (doi:10.1111/j.1398-9995. 2009.01940.x)

13 Kramkimel N, Sibon C, Le Beller C, Saiag P \& Mahe E. Bullous DRESS in a patient on strontium ranelate. Clinical and Experimental Dermatology 200934 e349-e350. (doi:10.1111/j.1365-2230. 2009.03302.x)

14 Pernicova I, Middleton ET \& Aye M. Rash, strontium ranelate and DRESS syndrome put into perspective. European Medicine Agency on the alert. Osteoporosis International 200819 1811-1812. (doi:10.1007/s00198-008-0734-8)

15 Netelenbos JC, Geusens PP, Ypma G \& Buijs SJ. Adherence and profile of non-persistence in patients treated for osteoporosis - a large-scale, long-term retrospective study in The Netherlands. Osteoporosis International 201122 1537-1546. (doi:10.1007/ s00198-010-1372-5) 
16 Rozenberg S, Lefever A, Kroll M, Vandromme J, Paesmans M \& Ham H. Prescription attitudes among gynecologists towards two particular risk factors of osteoporosis: the patient's age and her bone mineral density. Maturitas 199932 19-24. (doi:10.1016/ S0378-5122(99)00002-X)

17 Lo JC, Pressman AR, Omar MA \& Ettinger B. Persistence with weekly alendronate therapy among postmenopausal women. Osteoporosis International $2006 \quad 17$ 922-928. (doi:10.1007/ s00198-006-0085-2)

18 Clowes JA, Peel NF \& Eastell R. The impact of monitoring on adherence and persistence with antiresorptive treatment for postmenopausal osteoporosis: a randomized controlled trial. Journal of Clinical Endocrinology and Metabolism 200489 1117-1123. (doi:10. 1210/jc.2003-030501)

19 Delmas PD, Vrijens B, Eastell R, Roux C, Pols HA, Ringe JD, Grauer A, Cahall D \& Watts NB. Effect of monitoring bone turnover markers on persistence with risedronate treatment of postmenopausal osteoporosis. Journal of Clinical Endocrinology and Metabolism 200792 1296-1304. (doi:10.1210/jc.2006-1526)

20 Recker RR, Gallagher R \& MacCosbe PE. Effect of dosing frequency on bisphosphonate medication adherence in a large longitudinal cohort of women. Mayo Clinic Proceedings $2005 \mathbf{8 0} 856-861$. (doi:10.4065/80.7.856)

21 Cotté FE, Fardellone P, Mercier F, Gaudin AF \& Roux C. Adherence to monthly and weekly oral bisphosphonates in women with osteoporosis. Osteoporosis International $2010 \quad 21 \quad 145-155$. (doi:10.1007/s00198-009-0930-1)

22 Cooper A, Drake J \& Brankin E. Treatment persistence with oncemonthly ibandronate and patient support vs. once-weekly alendronate: results from the PERSIST study. International Journal of Clinical Practice 200660 896-905. (doi:10.1111/j. 1742-1241.2006.01059.x)

23 Weiss TW, Henderson SC, McHorney CA \& Cramer JA. Persistence across weekly and monthly bisphosphonates: analysis of US retail pharmacy prescription refills. Current Medical Research and Opinion 200723 2193-2203. (doi:10.1185/030079907X226069)
24 Penning-van Beest FJ, Erkens JA, Olson M \& Herings RM. Determinants of non-compliance with bisphosphonates in women with postmenopausal osteoporosis. Current Medical Research and Opinion 200824 1337-1344. (doi:10.1185/ 030079908X297358)

25 McClung M, Recker R, Miller P, Fiske D, Minkoff J, Kriegman A, Zhou W, Adera M \& Davis J. Intravenous zoledronic acid $5 \mathrm{mg}$ in the treatment of postmenopausal women with low bone density previously treated with alendronate. Bone $2007 \mathbf{4 1} 122-128$. (doi:10.1016/j.bone.2007.03.011)

26 Saag K, Lindsay R, Kriegman A, Beamer E \& Zhou W. A single zoledronic acid infusion reduces bone resorption markers more rapidly than weekly oral alendronate in postmenopausal women with low bone mineral density. Bone $2007 \mathbf{4 0} 1238-1243$. (doi:10.1016/j.bone.2007.01.016)

27 Schilcher J, Michaelsson K \& Aspenberg P. Bisphosphonate use and atypical fractures of the femoral shaft. New England Journal of Medicine $2011 \quad 364 \quad 1728-1737 . \quad$ (doi:10.1056/NEJMoa 1010650)

28 Shane E, Burr D, Ebeling PR, Abrahamsen B, Adler RA, Brown TD, Cheung AM, Cosman F, Curtis JR, Dell R, Dempster D, Einhorn TA, Genant HK, Geusens P, Klaushofer K, Koval K, Lane JM, McKiernan F, McKinney R, Ng A, Nieves J, O'Keefe R, Papapoulos S, Sen HT, van der Meulen MC, Weinstein RS \& Whyte M. Atypical subtrochanteric and diaphyseal femoral fractures: report of a task force of the American Society for Bone and Mineral Research. Journal of Bone and Mineral Research 2010 25 2267-2294. (doi:10.1002/jbmr.253)

Received 5 November 2011

Revised version received 7 January 2012

Accepted 18 January 2012 